\title{
O SOFRIMENTO MENTAL E O CUIDADO EM SAÚDE: PLANTAS MEDICINAIS E AS POLÍTICAS PÚBLICAS DE SAÚDE
}

\author{
Lauren Pieta Canan 1 \\ Jane Kelly Oliveira Friestino ${ }^{2}$ \\ Graciela Soares Fonsêca ${ }^{3}$ \\ Maria Eneida de Almeida 4
}

\section{Resumo}

O entendimento do sofrimento mental tomou formas diferentes no decorrer da história, ora por caracterizar-se como negativo e místico pelo viés religioso, ora por ser considerado algo que partia da vontade do sujeito. Atualmente, olha-se o sujeito em sofrimento mental não só por seus limites e fragilidades, mas como sujeito saudável, com potencialidades que podem exacerbar a capacidade do indivíduo que não está em sofrimento o tempo todo. Isto posto, compreende-se a necessidade de respeitar as escolhas de vida e a forma com que o sujeito relaciona-se com o meio que ocupa, ao mesmo tempo que pontua-se elementos que contribuem para o bem-estar físico, mental e social. Entendendo a importância do debate sobre o assunto, esse trabalho tem por objetivo apresentar o relato de experiência da atividade de extensão, intitulada: "O sofrimento mental e o cuidado em saúde: variáveis ambientais e as plantas medicinais", realizada por meio de uma extensão universitária junto ao Movimento das Mulheres Camponesas, que oportunizou um espaço de reflexão sobre o tema. A atividade foi desenvolvida no município de Palmitos/SC, no ano de 2017, durante o IV Encontro Municipal da Alimentação Saudável e oportunizou a troca entre o saber produzido na academia e o saber popular relacionado às plantas medicinais. Nesse encontro, foram ressaltados resultados significativos no que se refere ao campo da saúde mental e a integração do tratamento, agregado ainda ao uso de plantas medicinais.

Palavras-chave: Estresse psicológico. Extensão Universitária. Organização social. Plantas Medicinais

\footnotetext{
${ }^{1}$ Universidade Federal da Fronteira Sul campus Chapecó, Acadêmica do curso de Ciências Sociais. Membro do Núcleo de Pesquisa Território Ambiente e Paisagem- NETAP. E-mail: laurenpietacanan@gmail.com

2 Universidade Federal da Fronteira Sul campus Chapecó, Prof ${ }^{a}$ Doutora em Saúde Coletiva, Orientadora da Extensão

Universitária. Membro do Núcleo de Pesquisa Território Ambiente e Paisagem- NETAP. Email: jane.friestino@uffs.edu.br

${ }^{3}$ Universidade Federal da Fronteira Sul campus Chapecó, Prof ${ }^{a}$ Doutora em Ciências Odontológicas. Membro do Grupo de Pesquisa Políticas Públicas e Gestão em Saúde. E-mail: graciela.fonseca@uffs.edu.br

4 Universidade Federal da Fronteira Sul campus Chapecó, Prof ${ }^{a}$ Doutora em Saúde Coletiva. Membro do Grupo de Pesquisa Políticas Públicas e Gestão em Saúde. E-mail: maria.almeida@uffs.edu.br
} 


\begin{abstract}
The understanding of mental suffering took different forms in the course of history, sometimes to be characterized as negative and mystical by religious bias, sometimes because it was considered something that departed from the will of the subject. Nowadays, the subject is viewed in mental suffering not only because of its limits and fragilities, but also as a healthy subject with potentialities that can exacerbate the capacity of the individual who is not in suffering all the time. This fact includes the need to respect the life choices and the way in which the subject relates to the environment he occupies, while at the same time punctuating elements that contribute to the physical, mental and social well-being. Understanding the importance of the debate on the subject, this paper aims to present the experience report of the extension activity, entitled: "Mental suffering and health care: environmental variables and medicinal plants", carried out by means of an extension with the Peasant Women's Movement, which provided a space for reflection on the theme. The activity was developed in the municipality of Palmitos / SC, in the year 2017, during the IV Municipal Meeting of Healthy Eating and gave a chance to exchange between the knowledge produced in the academy and the popular knowledge related to medicinal plants. In this activity, significant results were highlighted regarding the field of mental health and the integration of treatment, added to the use of medicinal plants.
\end{abstract}

Keywords: Stress, Psychological. University Extension. Social organization. Plants, Medicinal

\title{
Introdução
}

A saúde mental no Brasil, ao longo de sua história, configurou-se por meio de indicações de indivíduos afetados por doenças/transtornos mentais que eram internados em hospitais psiquiátricos - antigos manicômios, e excluídos dos seus círculos sociais, ou seja da vida em sociedade (MOLINER; LOPES, 2013). Esse panorama começa a mudar com o início das discussões da Reforma Psiquiátrica, na década de 1980, e com a comutação da assistência extrahospitalar e não supressiva. Mas, o ápice dessa mudança preludia com a criação do Sistema Único de Saúde (SUS) por meio da Constituição Federal em 1988, quando foi instaurada, de fato, a formulação das políticas de saúde mental. Com a mudança nos modelo de internação e exclusão social para uma perspectiva de fortalecer a rede extra-hospitalar, consolidada com a já citada Reforma Psiquiátrica, e a criação do SUS; a Atenção Básica ganha lugar fundamental nesse processo.

Com isso, a Estratégia de Saúde da Família (ESF) principal modelo para organização da Atenção Básica no Brasil, configurou-se um espaço de práticas que fomentam novas perspectivas acerca do cuidado em saúde mental, no qual as concepções de: integralidade, intersetorialidade, territorialidade e interdisciplinaridade estruturaram esse novo modo de cuidar (AOSANI; NUNES, 2013). O novo panorama relacionado ao cuidado em saúde mental presente na Atenção Básica culminou ao surgimento de novas discussões, mudanças e dinamização do atendimento, resultando em um aumento na procura de respostas e ajudas nesse nível da atenção. O que se evidencia pelo elevado número de atendimentos que se refere à saúde mental na Atenção Básica (MOLINER; LOPES, 2013).

Sendo assim, ao compreender que as demandas de Saúde Mental encontram-se visíveis nas infinitas queixas manifestadas por parte dos usuários nos serviços de saúde, principalmente na Atenção Básica, e cientes da necessidade de discussões sobre o tema, um grupo de professores 
e acadêmicos vinculados à Universidade Federal da Fronteira Sul (UFFS) - campus Chapecó/SC, a fim de discutir e refletir sobre a saúde mental, desenvolveram a atividade de extensão intitulada “O sofrimento mental e o cuidado em Saúde: variáveis ambientais e as plantas medicinais". O convite para a realização da atividade proposta surgiu a partir de uma demanda apresentada pelo coletivo de mulheres pertencentes ao Movimento das Mulheres Camponesas (MMC) da cidade de Palmitos/SC.

A parceria com o MMC já havia acontecido em outras atividades. Isso porque a UFFS, campus Chapecó/SC é fruto da participação de sujeitos sociais, representantes da sociedade civil, e tem os movimentos sociais como fomento de construção da universidade, sendo apontada como uma das primeiras universidades advindas de um cenário de participação social e política (TREVISOL, 2015). E para que seja dada continuidade de participação popular, nos debates e nas discussões da universidade, a UFFS preocupa-se em estabelecer diálogos e parcerias com essas organizações.

Uma dessas parcerias, como já dito, é o MMC. É um movimento significativo para a região em que a UFFS está instalada, em especial na região Oeste de Santa Catarina. O movimento é oriundo dos diferentes movimentos sociais consolidados nos anos 1980, portando mais de três décadas de discussões e atividades. Passando de Movimento das Mulheres Agricultoras (MMA), para MMC em 2004, após a união de vários movimentos de mulheres do campo que compreendiam a importância da Via campesina como um espaço de exposição de ideias das mulheres agricultoras e fortalecimento das lutas por direitos. O surgimento do MMC em Santa Catarina viabilizou lutas frente aos direitos trabalhistas, a construção de uma identidade, a discussão de gênero por parte do movimento, e o engajamento com outras organizações sociais (BONI, 2013). O MMC, com o intuito da construção dessa identidade, realiza vários eventos durante o ano, e um deles é o evento municipal denominado: 'Dia da Alimentação Saudável'; em que o grupo da UFFS realizou a atividade sobre: "O sofrimento mental e o cuidado em Saúde: variáveis ambientais e as plantas medicinais"..

\section{Metodologia}

Trata-se de um relato de experiência vivenciado durante a atividade de extensão intitulada: "O sofrimento mental e o cuidado em Saúde: variáveis ambientais e as plantas medicinais", realizada no dia 08 de novembro de 2017, por professores e acadêmicos da UFFS, campus Chapecó/SC em conjunto com o MMC de Palmitos/SC. A realização da atividade de extensão junto ao MMC foi pensada partindo da trajetória entre os Movimentos Sociais e a Universidade.

Ao levar em conta esse processo de construção da UFFS e a importância do MMC, no cenário do Oeste Catarinense, preparou-se a já mencionada atividade de extensão em conjunto. A 
atividade foi desenvolvida através de um workshop durante o IV Encontro Municipal da Alimentação Saudável, oferecido gratuitamente, vinculando a educação acadêmica à perspectiva pedagógica da educação popular, fortalecendo a participação da comunidade acadêmica e regional nas atividades referentes ao ensino, pesquisa e extensão.

O principal objetivo da ação empreendida foi a promoção de saúde, utilizando-se da abordagem global do assunto: cuidado ao sofrimento mental. Durante a ação, preocupou-se em sistematizar o tema ao conjunto de saberes do grupo de mulheres do MMC - o cultivo das plantas medicinais. Entende-se que essa atividade, realizada pelo movimento, bem como a dinâmica presente no cultivo dos hortos comunitários, contribuem para a promoção da saúde, constituindose como importante fonte de matéria-prima; local para ação de educação em saúde e para a participação das pessoas no cultivo das espécies, destacando-se como um importante dispositivo terapêutico no campo da Saúde Mental (BRASIL, 2013).

Durante o evento, foram desenvolvidas duas oficinas que fizeram parte da atividade de extensão. A oficina 01: “Empoderamento, variáveis ambientais e o sofrimento mental” e Oficina 02: "Políticas públicas que reconhecem o uso e valorizam o conhecimento popular das plantas medicinais". Com o objetivo de oportunizar um espaço de reflexão referente ao sofrimento mental. Proporcionando a troca de saberes entre o coletivo das Mulheres Camponesas de Palmitos que realiza atividades práticas relacionadas às plantas medicinais e cultivo de hortos medicinais no município e os resultados parciais do grupo de pesquisa sofrimento mental na Atenção Básica da UFFS, campus Chapecó/SC.

A oficina 01 ocorreu em duas etapas e foi ministrada por duas docentes e uma discente, ambas da UFFS, durando cerca de trinta minutos. Utilizou-se a metodologia de roda de conversa e o objetivo da oficina foi pensar ferramentas para promoção da saúde através dos cuidados paliativos e a integração de elementos de apoio. Segundo elenca Fernandes (2009), esse fatores de apoio podem ser as atividades física, o lazer, principalmente, as atividades em grupo e o autoconhecimento.

Inicialmente, as pessoas foram convidadas a participar dizendo seu nome, bem como quem o escolheu e como ele foi escolhido. Esse primeiro momento contou com histórias que causaram comoção dos presentes, e risos ao mesmo tempo. Em seguida, foi proposto que os participantes pensassem na pessoa mais importante do mundo para eles. Após um rápido período de reflexão, uma caixa fechada passou de mão em mão, com a seguinte frase: “Quem é a pessoa mais importante do mundo para você?”. Depois de ler a pergunta, a caixa era aberta, e em seu interior havia outra frase dizendo: “Esta é a pessoa mais importante do mundo!". E abaixo estava um espelho.

Posteriormente, quando todos os presentes já haviam passado por esse processo, alguns questionamentos como: se elas haviam pensado que eles eram as pessoas mais importantes do 
mundo e, se não, porque isso aconteceu? Obteve-se os mais diversos tipos de respostas. Mas, a principal colocação foi a de que, geralmente, não pensamos em nós em primeiro lugar. Através dessa estratégia, foram iniciadas as discussões sobre o tema do sofrimento mental, pontuando os aspectos do autocuidado; do entendimento do que é estar em sofrimento; como e quando procurar ajuda; e se a saúde mental era debatida por eles.

A oficina 02 teve como objetivo central divulgar as políticas públicas que estão vinculadas ao reconhecimento, valorização e uso das plantas medicinais pela população, com incentivo oficial do Ministério da Saúde. A metodologia foi uma roda de conversa com a intenção de aproximar os presentes, que foram questionados sobre o conhecimento relacionado às políticas públicas que envolvem as plantas medicinais. De maneira geral, foi uma surpresa, visto que a maioria não sabia do que se tratava. E foi nesse sentido que foi observada a importância de disponibilizar essas informações, pois muitas intervenções podem ser viabilizadas ao se conhecer os caminhos para as ações. As duas oficinas ocorreram ao mesmo tempo, sendo os presentes divididos em dois grupos, e cada grupo permaneceu 30 minutos em cada estação, ocorrendo um rodízio.

A avaliação do workshop aconteceu através da participação dos presentes e suas interações com as atividades propostas. Assim como a observação com o engajamento na discussão do tema, através de perguntas abertas que buscavam saber se as atividades haviam suprido as expectativas dos participantes, que disponibilizaram um momento de seu tempo para participar. Por fim, ocorreu uma devolutiva por parte de representantes do MMC sobre a ação e reação das práticas desenvolvidas.

\section{Resultados}

Durante o dia de atividades desenvolvidas em Palmitos/SC, participaram aproximadamente 100 pessoas, em maior número mulheres na faixa etária de 59 anos. Dentre o público, a maioria era da cidade de Palmitos/SC, mas também haviam pessoas de municípios vizinhos. Vale ressaltar que o município de Palmitos/SC tem como características ser uma cidade de pequeno porte, com uma população estimada de aproximadamente 17 mil habitantes, fazendo fronteira com o Rio Grande do Sul, distante $60 \mathrm{~km}$ do campus Chapecó da UFFS. Sua principal atividade econômica é agrícola, em especial a agricultura familiar (FRIESTINO et al, 2016).

O workshop foi acolhido com muito entusiasmo e motivação por parte dos presentes. As oficinas 01 e 02, apesar de partirem de dinâmicas diferentes, incitaram a discussão sobre o sofrimento mental, a necessidade do cuidado e do autoconhecimento, e de estudos e aprimoramentos na Atenção Básica em saúde.

A oficina 01 trouxe diversos relatos de pessoas que viviam em sofrimento, que 
conheciam alguém nesse estado, além de falas de participantes que sentiam-se incomodados, na maioria das vezes, em tocar no assunto. As etapas desenvolvidas neste movimento contaram com a preocupação de levar um diálogo que possibilitasse uma compreensão fácil, capaz de envolver o público, e estimular a participação dos presentes. Os relatos obtidos durante a atividade, junto da vivência compartilhada entre os presentes, permitiram observar seus entendimentos sobre o sofrimento mental, além da clareza de que esse tema precisa ser discutido, porém ainda é atrelado a muitos estigmas e preconceitos (BRASIL, 2013).

Ficou visível, por intermédio dos exercícios propostos, que dialogar sobre saúde mental não constitui um hábito rotineiro, ficando restrito aos consultórios dos profissionais de saúde. Evidenciou-se a necessidade de desvelar o estigma da população sobre a condição de um sujeito em sofrimento, sensibilizando as pessoas sobre como os preconceitos podem ser agravantes na procura por um tratamento adequado. Ao levar isso em conta, compreende-se que esse papel é dever da população como um todo, sejam profissionais da saúde ou não. Mas a Atenção Básica, em especial, tem função significativa na redução de estigmas, possibilitando a administração de muitas situações (BRASIL, 2013).

A oficina 02 teve como objetivo central divulgar as políticas públicas que estão vinculadas ao reconhecimento, a valorização e ao uso das plantas medicinais pela população, com incentivo do Ministério da Saúde. A roda de conversa executada teve como princípio aproximar os presentes. Inicialmente perguntou-se quem deles tinha conhecimento das políticas públicas na área da saúde que envolvem as plantas medicinais. A primeira reação foi de surpresa, visto que a maioria não tinha ideia do que se tratava, do que estava sendo perguntado. E foi nesse sentido que foi observada a importância de disponibilizar essas informações, pois muitas ações podem ser viabilizadas ao se conhecer os caminhos oficiais.

A partir dessa constatação, foram apresentadas duas políticas lançadas em 2006, cujo desenvolvimento nacional tem fomentado o interesse neste campo do saber e de práticas populares: a) a Política Nacional de plantas Medicinais e Fitoterapia (BRASIL, 2006a) e b) a Política Nacional de Práticas Integrativas e Complementares (BRASIL, 2006b). Em que, as Plantas Medicinais têm uma trajetória relevante em todo o Brasil, com a construção de Hortos Medicinais e de Farmácias Vivas na Atenção Básica do SUS (BRASIL, 2012). Ela propõe a inclusão das plantas medicinais e fitoterapia, homeopatia, medicina tradicional chinesa/acupuntura e termalismo social/crenoterapia como opções terapêuticas no sistema público de saúde.

Essa política traz, entre suas diretrizes para plantas medicinais e fitoterapia, a elaboração da Relação Nacional de Plantas Medicinais e de Fitoterápicos; e o provimento do acesso a plantas medicinais e fitoterápicos aos usuários do SUS. O avanço nesta área do conhecimento é demonstrado pelo lançamento do Programa Nacional de Plantas Medicinais e Fitoterápicos 
(BRASIL, 2016). Esse programa é proveniente dessas políticas que visam a fortalecer as ações no SUS, para a melhoria do acesso da população aos medicamentos, à inclusão social e regional, ao desenvolvimento industrial e tecnológico, além do uso sustentável da biodiversidade brasileira e da valorização, da valoração e preservação do conhecimento tradicional associado das comunidades tradicionais e indígenas.

Todavia, durante as duas atividades foi possível contemplar os mesmos desafios enfrentados por profissionais na Atenção Básica. Tornou-se possível identificar nos discursos dos participantes o que Moliner e Lopes (2013) constataram também em sua pesquisa. Segundo elas, a noção de saúde mental perpassa o conceito de saúde igual à doença, sendo esse ainda um conceito biologista e objetivo, sem olhar para as questões subjetivas e a dimensão social que envolve os usuários, e além da concepção é necessária a prática que responda a esse novo olhar. A falta desse novo olhar, na maioria das vezes, ignora a questão social que envolvem o sujeito em sofrimento, que além de precisar lidar com a compreensão e feitos da sua condição é pressionando a desenvolver mecanismos de defesa, a fim de cumprir seu papel social quanto status social que ocupa e resistir aos estigmas de sua condição de sujeito em sofrimento mental.

\section{Discussão}

As atividades de extensão, quando dialogam com o Ensino e a Pesquisa, tendem a atender a demandas da sociedade, fugindo de um caráter assistencialista das primeiras extensões universitárias, e fortalecendo o vínculo com a comunidade externa, proporcionando atividades que reflitam no cenário e proporcionam modificações e afirmações dos meios envolvidos. Segundo o documento redigido na I Conferência de Ensino Pesquisa e Extensão (COEPE) da UFFS, é através dessa nova concepção de atividade de extensão que a população é entendida como sujeito agente e faz parte do processo de conhecimento como um todo. Subentende-se, nesse processo, a articulação do saber acadêmico para com o saber popular e instaura (UFFS, 2011).

A relação estabelecida entre a sociedade e a universidade encontra-se assinalada pela troca de saberes, que supera o discurso de preponderância acadêmica, e torna possível a convenção entre grupos distintos, assim como o laço com movimentos sociais (UFFS, 2011). Mediante isso, entende-se que a participação dos movimentos é fundamental nesse processo, uma vez que os mesmos constroem ações deliberadas através do meio em que estão instaurados, promovendo a construção de um cenário baseado na cidadania, o que também proporciona a afirmação e mudanças esperadas.

Ao levar em conta a atividade desenvolvida, é válido ressaltar também a importância do workshop para a acadêmica envolvida na atividade. O espaço da ação proporcionou um ambiente de reflexão e inovação de conhecimento, através da extensão universitária desenvolvida com o MMC na qual a graduanda adentra a comunidade e sai dos "muros" da universidade. Neste 
momento, ocorre a aproximação com os sujeitos, permitindo conhecer a realidade do grupo envolvido, bem como analisar a pertinência das atividades desenvolvidas como ação de prevenção e promoção da saúde (RODRIGUES, et al., 2013). Diante disso, percebe-se que o workshop ofertou além de um ambiente de trocas, como já citado, novos panoramas à acadêmica, fornecendo possibilidades de uma formação integral e interdisciplinar.

A UFFS estrutura esses entendimentos, acima citados em sua política de extensão, visando garantir a extensão universitária como percurso cultural, científico e educativo, a fim de promover uma transformação entre a relação Universidade e Sociedade, fomentando atividades como a que aqui vem sendo relatada, através da troca de saberes, da democratização, da interdisciplinaridade e da produção do conhecimento acadêmico (UFFS, 2011).

Desta forma, o workshop desenvolvido apresentou-se como um espaço potente para o fortalecimento das ações voltadas as atividades de extensão, assim como ao campo da saúde mental e das plantas medicinais. A atividade oportunizou a integração dos mais diversos saberes, articulando, como já descrito, os saberes acadêmicos e sociais. Além de oportunizar a reflexão sobre a discussão da saúde mental, tanto para os participantes quanto para os professores e acadêmicos que promoveram as ações no evento. Consolidou-se por meio do movimento proposto e pela troca de saberes, a necessidade de trabalhar os estigmas sobre a saúde mental presentes no cotidiano dos sujeitos; assim como a necessidade da formação permanente a fïm de estimular elaboração de ações que sirvam como ferramentas para uma atuação mais inclusiva desses sujeitos em sofrimento (BRASIL, 2013).

\section{Considerações Finais}

Sabendo que o sofrimento mental é responsável por levar os indivíduos à perdas significativas em seu cotidiano, julgou-se necessário, comprovando que o mesmo é uma pauta importante a ser tratada na realidade das mulheres que fazem parte desse movimento social. Desta forma, o espaço de discussão, proporcionado pela atividade de extensão, foi capaz de evidenciar aspectos do sofrimento mental nas atividades diárias dos indivíduos, assim como os estigmas e preconceitos acerca do tema. O workshop também contribuiu para fortalecer o vínculo da Universidade com o MMC, oportunizando um espaço de trocas e experiências significativas.

Através das ações desenvolvidas percebeu-se a necessidade de olhar para a saúde mental para além de um fator biológico. É de fundamental importância empregar um novo olhar para a pessoa em sofrimento, a fim de entender que a condição do sujeito também advém de fatores sociais e culturais dominantes ao longo da construção histórica de nossa sociedade. Entre os depoimentos dos participantes durante as oficinas. Visualizou-se a participação no movimento como fator de apoio contribuinte para manutenção de uma vida saudável. As falas traziam elementos de apoio para o grupo e retratavam a segurança de estar se expondo em meio a aquele 
grupo.

Frente ao que foi encontrado durante a atividade, observa-se a relevância das discussões sobre a saúde mental para além das edificações dos serviços de saúde e dos fatores biológicos. A utilização das plantas medicinais, por exemplo, como conteúdo para a discussão do tema foi extremamente relevante. E capaz de tratar uma temática estigmatizada a partir do conhecimento das participantes daquele grupo, posicionando o deslocamento da ideia do que seria o sujeito em sofrimento, mas englobando a realidade do movimento para com as discussões. A discussão do tema, desta forma, deve levar em conta o cotidiano dos sujeitos, e dos lugares que estes ocupam através do trabalho, dos estudos, das relações de parentesco, do lazer e da construção da sua história.

\section{Agradecimentos:}

Agradecemos ao coletivo de mulheres do Movimento das Mulheres Camponesas de Palmitos-SC pela infraestrutura fornecida; além de toda comunidade participante das atividades realizadas.

\section{Referências}

AOSANI, T. R.; NUNES, K. G. A Saúde Mental na Atenção Básica: A percepção dos Profissionais de Saúde. Revista Psicologia e Saúde, Porto Alegre: v. 5, n. 2, jul. /dez. 2013, p. $71-80$

BONI, V. Movimento de mulheres camponesas: um movimento camponês e feminista. Revista Grifos, Chapecó: v.22, n.34/35, 2013.

BRASIL. Ministério da Saúde. Secretaria de Ciência, Tecnologia e Insumos Estratégicos. Departamento de Assistência Farmacêutica. Política nacional de plantas medicinais e fitoterápicos. Brasília: Ministério da Saúde, 2006a.

Ministério da Saúde. Secretaria de Atenção à Saúde. Departamento de Atenção Básica. Política Nacional de Práticas Integrativas e Complementares no SUS - PNPICSUS. Brasília : Ministério da Saúde, 2006b.

Ministério da Saúde. Secretaria de Atenção à Saúde. Departamento de Atenção Básica. Cadernos de Atenção Básica no 31. Práticas integrativas e complementares: plantas medicinais e fitoterapia na Atenção Básica. 2012. Disponível em:

<http://189.28.128.100/dab/docs/publicacoes/geral/miolo_CAP_31.pdf >. Acesso em: 23 jun. 2018.

- Ministério da Saúde. Secretaria de Atenção à Saúde. Departamento de Atenção Básica. Cadernos de Atenção Básica no 34 Saúde Mental, 2013. Disponível em: http://bvsms.saude.gov.br/bvs/publicacoes/cadernos_atencao_basica_34_saude_mental.pdf . Acesso em: 23 jun 2018.

Ministério da Saúde. Secretaria de Ciência, Tecnologia e Insumos 
de Plantas Medicinais e Fitoterápicos. Brasília: Ministério da Saúde, 2016.

FERNANDES, W. J. Saúde mental: uma visão vincular. Revista da SPAGESP - Sociedade de Psicoterapias Analíticas Grupais do Estado de São Paulo, Jul.-Dez. 2009, v. 10, n. 2, p. 2232.

FRIESTINO, JKO; SILVA, OMP; NASCIMENTO, MC. Condições sanitárias no Oeste de Santa Catarina: construindo um diagnóstico das populações rurais e pequenas comunidades. Rev. bras. ciên. saúde. 2016, v.19, n.2, p. 109-116, 2015.

MOLINER, J.; LOPES, S. M. B. Saúde mental na atenção básica: possibilidades para uma prática voltada para a ampliação e integralidade da saúde mental. Saúde Soc: São Paulo, v.22, n.4, 2013, p.1072-1083.

RODRIGUES, A.L.; et al. Contribuições da Extensão Universitária na Sociedade. Cadernos de Graduação - Ciências Humanas e Sociais:| Aracaju, v. 1, n.16, mar. 2013.

TREVISOL, J. A pós-graduação na Universidade Federal da Fronteira Sul: interiorização e redução de assimetrias em uma Região de fronteira. RBPG, Brasília, v. 12, n. 28, p. 505-532, ago. 2015.

UFFS. Universidade Federal da Fronteira Sul. Construindo agendas e definindo rumos: I Conferência de Ensino, pesquisa e extensão da UFFS / Universidade Federal da Fronteira Sul ; organizadores: Joviles Vitório Trevisol, Maria Helena Cordeiro e Monica Hass. Chapecó : UFFS, 2011. 